\title{
Physical implications of Fisher-information's scaling symmetry
}

Research Article

\author{
Silvina P. Flego ${ }^{1}$, Angelo Plastino $2,4,5 *$, Angel R. Plastino $0^{3,4}$
}

1 Universidad Nacional de La Plata, Facultad de Ingeniería,

Grupo de Investigación Teórica y Aplicada en Teoría de la Información (GTyATI), 1900 La Plata, Argentina.

2 Universidad Nacional de La Plata, Instituto de Física (IFLP-CCT-CONICET),

C.C. 727,1900 La Plata, Argentina

3 CREG-Universidad Nacional de La Plata-CONICET,

C.C. 727,1900 La Plata, Argentina

4 Instituto Carlos I de Fisica Teorica y Computacional and Departamento de Fisica Atomica, Molecular y Nuclear, Universidad de Granada, Granada, Spain

5 Universitat de les IIles Balears and IFISC-CSIC, 07122 Palma de Mallorca, Spain

Received 26 September 2011 ; accepted 15 January 2012

\begin{abstract}
:
We study the scaling properties of Fisher's information measure (FIM) and show that from these one can straightforwardly deduce significant quantum-mechanical results. Specifically, we investigate the scaling properties of Fisher's measure $/$ and encounter that, from the concomitant operating rules, several interesting, albeit known, results can be derived. This entails that such results can be regarded as pre-configured by the conjunction of scaling and information theory. The central notion to be arrived at is that scaling entails that I must obey a certain partial differential equation (PDE). These PDE-solutions have properties that enable the application of a Legendre-transform (LT). The conjunction PDE+LT leads one to obtain several quantum results without recourse to the Schrödinger's equation.
\end{abstract}

PACS (2008): 03.65.-w, 03.65.Ta, 05.70.-a, 05.90.+m, 87.19.10

Keywords: $\quad$ scaling transform $•$ Fisher Information $\bullet$ Legendre structure $\cdot$ reciprocity relations

(c) Versita Sp. z o.o.

\section{Introduction}

In this communication we wish to show that from the scaletransformation rules obeyed by Fisher's information mea-

*E-mail: angeloplastino@gmail.com sure (FIM) some interesting quantum results immediately follow.

\subsection{Scaling}

Symmetry in physics means invariance, i.e. lack of change under some transformation, e.g. coordinate transformations. The concept is one of the most powerful tools of 
physics as practically all laws of nature originate in symmetries. Anderson wrote in 1972 that "it is only slightly overstating the case to say that physics is the study of symmetry" [1]. Here we will concern ourselves with scaling symmetry, that refers to the idea that if a system is expanded or reduced in size, the transformed system exhibits the same properties as the original. This symmetry is notable for the fact that it does not exist for most physical systems.

Thus, scale invariance is a feature of objects or laws that do not change if scales of length, energy, or other variables, are multiplied by a common factor. A technical term for this transformation is "dilatation" (or dilation). The dilatations can also form part of a larger conformal symmetry. It is possible for the probability distributions of random processes to display this kind of scale invariance. In classical field theory, scale invariance most commonly applies to the invariance of a whole theory under dilatations. In quantum field theory, scale invariance has an interpretation in terms of particle physics. In a scale-invariant theory, the strength of particle interactions does not depend on the energy of the particles involved. In statistical mechanics, scale invariance is a feature of phase transitions. The key observation is that near a phase transition or critical point, fluctuations occur at all length scales, and thus one should look for an explicitly scale-invariant theory to describe the phenomena. Such theories are scaleinvariant statistical field theories, and are formally very similar to scale-invariant quantum field theories [2].

\subsection{Fisher's information}

Physical applications of Fisher's information measure (FIM) have grown exponentially in the last two decades (see $[3,4,6]$ and the vast references therein). A very restricted list of papers is given in [7-13].

A very common Fisher-technique replaces Shannons' entropy by FIM in applications of the celebrated Jaynes' MaxEnt technique $[15,16]$. We do not require employment of such technique here.

\subsection{Our program}

We review in Sec. 2 some fundamentals of the FIM, denoted by $I$. In Sec. 3 we investigate I's behavior under scale-changes. From the concomitant scaling rule we derive in Sec. 4 a partial differential equation for I. In Sec. 5 a Legendre transform (LT) is applied to its solution and we find that some quantum results immediately emerge (for instance, the virial theorem). Such procedure allows, now in Sec. 6, to derive the scaling-behavior of the eigenvalues of Schrödinger's equation. Finally, some conclusions are drawn in Sec. 7.

\section{Fisher's information measure (FIM)}

FIM arises as a measure of the expected error in a measurement [4]. We restrict here ourselves just to a particular but quite significant FIM-case, that of translation families [4, 6-10], i.e. distribution whose form does not change under displacements of a shift parameter. The ensuing distributions are shift-invariant -à la Mach, no absolute origin- and the ensuing FIM obeys Galilean invariance [4]. This FIM-type refers to translations of an onedimensional observable $x$ with probability density $f(x)$. It reads, setting first $f=\psi^{2}$ for a real amplitude $\psi$ [11]

$$
I=\int \mathrm{d} x f(x)\left(\frac{\partial \ln f(x)}{\partial x}\right)^{2}=4 \int \mathrm{d} x\left(\frac{\partial \psi(x)}{\partial x}\right)^{2}
$$

The associated Cramer-Rao bound $[4,11,17]$ is the inequality

$$
l e^{2} \geq 1
$$

where $e$ is the variance of the stochastic variable $x$ [11]

$$
e^{2}=\int \mathrm{d} x f(x) x^{2}-\left(\int \mathrm{d} x f(x) x\right)^{2},
$$

representing the mean-square error associated to the pertinent measurement.

\subsection{The independent variables on which / de- pends}

Now focus on a stochastic system specified by a set of $M$ physical parameters $\mu_{k}$, of the form $\mu_{k}=\left\langle A_{k}\right\rangle$, with $A_{k}=$ $A_{k}(x)$. The set of $\mu_{k}$-values is taken to represent some available, accessible empirical information out of which one constructs I, via a MaxEnt procedure [15], for instance. We choose here not to do so.

If the probability distribution function (PDF) that adequately describes the system is $f(x)$, it has to reproduce the known expectation values, i.e.

$$
\left\langle A_{k}\right\rangle=\int d x A_{k}(x) f(x), \quad k=1, \ldots, M .
$$

Also, one has, of course, a normalization condition

$$
\int d x f(x)=1
$$

Notice however that we are not claiming here that I exhibit any maximal or minimal character. 


\subsection{The scenario we are working with}

We put forward at this point the details of the scenario we are here contemplating.

- A Hilbert space is separable if and only if it has a countable orthonormal basis (labelled by integer numbers $k$ ). In particular, $\mathcal{L}_{2}$ is one such space.

- A particularly convenient basis we use is $1, x, x^{2}, x^{3}, \ldots, x^{n}, \ldots$ ("power-basis"). Remember that all basis are equivalent.

- We take it that the $A_{k}(x), f, \psi$, and any other function we might use here (generically called $U(x)$ ) belong to $\mathcal{L}_{2}$. Thus, any $A_{k}(x)$ or $U(x)$ admits of a series expansion in the power-basis [19]. This enables us to base our considerations on the assumption that the a priori knowledge refers to moments $x^{k}$ of the independent variable, i.e.

$\left\langle A_{k}\right\rangle=\left\langle x^{k}\right\rangle=\int d x x^{k} f(x) ; k=1, \ldots, M$,

and that one possesses information about $M$ of these moments $\left\langle x^{k}\right\rangle$.

- Eq. (1) can be recast, after integration by parts, in the fashion

$$
I=-4 \int \mathrm{d} x \psi(x) \frac{\partial^{2} \psi(x)}{\partial x^{2}}=-4\left\langle\frac{\partial^{2}}{\partial x^{2}}\right\rangle_{\psi}
$$

- We will actually use below the arbitrary function $U(x) \in \mathcal{L}_{2}$ and assume that the first $M$ terms of its basis-expansion suffice to represent it with whatever precision one might require. Consequently, we are enabled to cast $U(x)$ in the fashion

$$
U(x)=\frac{1}{8} \sum_{k}^{M} c_{k} x^{k}
$$

where the constants $c_{k}$ remain unspecified at this stage, since $U$ is arbitrary. The set

$$
\left\{c_{1}, c_{2}, \ldots, c_{M}\right\}
$$

will play an important role in future considerations.

- I is a function of the known mean values only, i.e.

$$
I=I\left(\left\langle x^{1}\right\rangle, \ldots,\left\langle x^{M}\right\rangle\right)
$$

\section{Scaling transform and FIM}

In a scaling transformation by a constant "length" $a \geq 0$ we have $x \rightarrow a x$. Remember now (10) and consider that under the scaling transformation (ST) of the theory the FIM / goes over to an equivalent ST-FIM $\bar{l}$, given by

$$
\bar{l}=\int \mathrm{d} x \bar{f}(x)\left(\frac{\partial \ln \bar{f}(x)}{\partial x}\right)^{2}
$$

where $\bar{f}$ is the pertinent ST-PDF. Now, the system is specified by a set of $M$ physical parameters $\bar{\mu}_{k}=\left\langle x^{k}\right\rangle_{T}$ which are to be regarded as our prior knowledge,

$$
\left\langle x^{k}\right\rangle_{T} \equiv \int d x x^{k} \bar{f}(x), \quad k=1, \ldots, M
$$

The relation between the PDF and the ST-PDF under the scaling transformation, is given by

$$
\bar{f}(x)=N f(a x)
$$

with $N$ fixed requiring that both PDF be normalized to unity

$$
1=\int \bar{f}(x) d x=\int N f(a x) d x=\frac{N}{a} \int f(u) d u
$$

entailing $N=a$. The scaling transformed moments (12) are given by

$$
\begin{aligned}
\left\langle x^{k}\right\rangle_{T} & =\int x^{k} \bar{f}(x) d x=a \int x^{k} f(a x) d x \\
& =a^{-k} \int x^{k} f(x) d x=a^{-k}\left\langle x^{k}\right\rangle,
\end{aligned}
$$

and the scaling transformed FIM $\bar{l}$ can be obtained from I as

$$
\begin{aligned}
\bar{I} & =\int \bar{f}(x)\left(\frac{\partial \ln \bar{f}(x)}{\partial x}\right)^{2} d x= \\
& =\int a f(a x)\left(\frac{\partial \ln [a f(a x)]}{\partial x}\right)^{2} d x= \\
& =a^{2} \int f(x)\left(\frac{\partial \ln f(x)}{\partial x}\right)^{2} d x=a^{2} I
\end{aligned}
$$

The above simple relation rules the FIM-behavior under a change of scale. 


\section{Equations governing scaling}

We start presenting our original results at this stage, invoking again (10). The FIM / under the scaling transformation $x \rightarrow a x$, goes over to an equivalent FIM $\bar{l}$ satisfying the ST-relation (16). In somewhat more detail one has $\left[\right.$ with $\left.\left\langle x^{k}\right\rangle_{T}=a^{-k}\left\langle x^{k}\right\rangle\right]$,

$$
a^{2} I\left(\left\langle x^{1}\right\rangle_{,} \cdots,\left\langle x^{M}\right\rangle\right)=\bar{I}\left(\left\langle x^{1}\right\rangle_{T}, \cdots,\left\langle x^{M}\right\rangle_{T}\right)
$$

Differentiating relation (17) with respect to the $a$-parameter we obtain

$$
\frac{\partial}{\partial a}\left[a^{2} I\left(\left\langle x^{1}\right\rangle, \cdots,\left\langle x^{M}\right\rangle\right)\right]=\frac{\partial}{\partial a}\left[\bar{l}\left(\left\langle x^{1}\right\rangle_{T}, \cdots,\left\langle x^{M}\right\rangle_{T}\right)\right],
$$

i.e.

$$
2 a I=\sum_{k=1}^{M} \frac{\partial \bar{l}}{\partial\left\langle x^{k}\right\rangle_{T}} \frac{\partial\left\langle x^{k}\right\rangle_{T}}{\partial a} .
$$

Note that

$$
\frac{\partial\left\langle x^{k}\right\rangle_{T}}{\partial a}=\frac{\partial}{\partial a}\left(a^{-k}\left\langle x^{k}\right\rangle\right)=-k a^{-1}\left\langle x^{k}\right\rangle_{T},
$$

entailing

$$
2 a I=-a^{-1} \sum_{k=1}^{M} k\left\langle x^{k}\right\rangle_{T} \frac{\partial \bar{I}}{\partial\left\langle x^{k}\right\rangle_{T}},
$$

or

$$
a^{2} I=-\sum_{k=1}^{M} \frac{k}{2}\left\langle x^{k}\right\rangle_{T} \frac{\partial \bar{l}}{\partial\left\langle x^{k}\right\rangle_{T}} .
$$

Using the ST-equalities (15) and (17) we arrive then to a linear, partial differential equation (PDE) for $I$,

$$
I=-\sum_{k=1}^{M} \frac{k}{2}\left\langle x^{k}\right\rangle \frac{\partial I}{\partial\left\langle x^{k}\right\rangle}
$$

The above is an important relation that describes FIM's behavior with respect to the scaling transform of the mean values that represent our prior knowledge. A similar equation was derived in [20], but for a totally different, MaxEntI-extremal Fisher-scenario [21]. The counterpart of (22) was derived in [20] starting from Schrödinger's equation (SE) and using:
1. the virial theorem plus

2. properties of a Legendre transform (LT) of the SE.

A detailed mathematical discussion of this partial differential equation (22) is available, including its so-called complete solution [20]. One finds from such considerations

$$
I\left(\left\langle x^{1}\right\rangle, \ldots,\left\langle x^{M}\right\rangle\right)=\sum_{k=1}^{M} A_{k}\left|\left\langle x^{k}\right\rangle\right|^{-2 / k}
$$

where $A_{k}$ are positive real numbers (integration constants)

- The $I$ - domain is

$$
D_{l}=\left\{\left(\left\langle x^{1}\right\rangle, \ldots,\left\langle x^{M}\right\rangle\right) \mid\left\langle x^{k}\right\rangle \in \Re_{o}\right\} .
$$

- Eq. (23) states that for $\left\langle x^{k}\right\rangle>0, I$ is a monotonically decreasing function of $\left\langle x^{k}\right\rangle$ [20].

- As one expects from a "good" information measure [4], $I$ is a convex function [20].

- The general solution for the I-PDE does indeed exist [20].

- Uniqueness can be proved from an analysis of the associated Cauchy problem by following the same procedure employed in Ref. [20]. In our present situation, the Lipschitz condition can be seen to be always verified since we can argue that, from a physics' stand-point, no amount of information can experience an abrupt, infinite change whenever a physical measurements suffers a small variation. Thus,

$$
\frac{\partial I}{\partial\left\langle x^{k}\right\rangle}<\infty
$$

\section{Changing independent variables from the $\left\langle x^{k}\right\rangle$ to the $c_{k}$ of Eq. (9)}

The LT is an operation that transforms one real-valued function of a real variable into another. Specifically, the LT of a convex (or concave) function $f$ is the function $f_{L}$ defined by [22]

$$
f_{L}(p)=\sup _{x}[p x-f(x)]
$$


If $f$ is differentiable, then $f_{L}(p)$ can be interpreted as the negative of the $y$-intercept of the tangent line to the graph of $f$ that has slope $p$. In particular, the value of $x$ that attains the maximum has the property that

$$
f^{\prime}(x)=p, \text { called a "reciprocity relation". }
$$

That is, the derivative of the function $f$ becomes the argument to the function $f_{L}$. In particular, if $f$ is convex (or concave), then $f_{L}$ satisfies the functional equation

$$
f_{L}\left(f^{\prime}(x)\right)=x f^{\prime}(x)-f(x) .
$$

The LT is its own inverse. Like the familiar Fourier transform, the LT takes a function $f(x)$ and produces a function of a different variable $p$. However, while the Fourier transform consists of an integration with a kernel, the LT uses maximization as the transformation procedure. The functional relationship specified by $f(x)$ can be represented equally well as a set of $(x, y)$ points, or as a set of tangent lines specified by their slope and intercept values.

FIM expresses a relation between the independent variables or control variables (the mean values) and the function $I$. Such information is encoded into the functional form of

$$
I=I\left(\left\langle x^{1}\right\rangle, \ldots,\left\langle x^{M}\right\rangle\right)
$$

For later convenience, we will also denote such a relation or encoding as $\left\{I,\left\langle x^{k}\right\rangle\right\}$. FIM's properties enable us to introduce the LT of $F=-l$, to be called $\alpha$, which is going to be a function depending on a new set of independent variables $c_{k}$ [cf. Eq. (9)]. We are changing independent variables from the $\left\{\left\langle x^{k}\right\rangle\right\}$ to the $\left\{c_{k}\right\}$.

This is accomplished via the LT [22]

$$
\alpha\left(c_{1}, \cdots, c_{M}\right)=\sum_{k=1}^{M} c_{k}\left\langle x^{k}\right\rangle-F
$$

or,

$$
\alpha=I+\sum_{k=1}^{M} c_{k}\left\langle x^{k}\right\rangle
$$

with the associated reciprocity relation [22]

$$
c_{k} \equiv \frac{\partial F}{\partial\left\langle x^{k}\right\rangle}=-\frac{\partial l}{\partial\left\langle x^{k}\right\rangle}
$$

At this point, from purely formal reasons, one should be able to guarantee that $\partial I / \partial\left\langle x^{k}\right\rangle<\infty$, as demonstrated in relation to Eq. (24), so as to legitimately claim that a LT $\alpha$ of FIM can be constructed. For appropriately dealing with $\alpha$ we would face the F-function [22]

$$
F\left(\left\langle x^{1}\right\rangle, \cdots,\left\langle x^{M}\right\rangle\right)=\sum_{k=1}^{M} c_{k}\left\langle x^{k}\right\rangle-\alpha
$$

or

$$
I=\alpha-\sum_{k=1}^{M} c_{k}\left\langle x^{k}\right\rangle
$$

with the associated "reciprocity relation" [22]

$$
\left\langle x^{k}\right\rangle \equiv \frac{\partial \alpha}{\partial c_{k}}
$$

We see that the LT-structure of FIM displays the information encoded in $I$, via $c_{k}$-parameters, in $\alpha=\alpha\left(c_{1}, \ldots c_{M}\right)$,

$$
\left\{I,\left\langle x^{k}\right\rangle\right\} \quad \stackrel{L T}{\longleftrightarrow} \quad\left\{\alpha, c_{k}\right\}
$$

Using (22) and (29) we can now write

$$
I=\sum_{k=1}^{M} \frac{k}{2} c_{k}\left\langle x^{k}\right\rangle
$$

and the $c_{k}$-parameters can be obtained via (23) and (29)

$$
c_{k}=-\frac{\partial I}{\partial\left\langle x^{k}\right\rangle}=\frac{2}{k} A_{k}\left|\left\langle x^{k}\right\rangle\right|^{-(2+k) / k}
$$

Substituting (33) in (28), the FIM-LT reads

$$
\alpha=\sum_{k=1}^{M} \frac{k+2}{2} c_{k}\left\langle x^{k}\right\rangle
$$

Then, from (35) and taking into account (32), we are led to a partial differential equation underlying the $\alpha$-function

$$
\alpha=\sum_{k=1}^{M} \frac{k+2}{2} c_{k} \frac{\partial \alpha}{\partial c_{k}}
$$

The above equation was first derived from a constrained Fisher variational problem in [23]. We are not appealing to this equation here but only to the FIM scaling symmetry 
instead. A complete solution for $\alpha$-PDE (36) is given by [23],

$$
\alpha\left(c_{1}, \ldots, c_{M}\right)=\sum_{k=1}^{M} B_{k}\left|c_{k}\right|^{2 /(2+k)}
$$

where the $B_{k}$ s are positive real numbers (integration constants). Eq.(37) states that for $c_{k}>0, \alpha$ is a monotonically increasing function of the $c_{k}$, and as one expect from the LT of $I$, we end up with a concave function. We may obtain the $\left\langle x^{k}\right\rangle$ 's from the reciprocity relations (32). For $c_{k}>0$ one gets

$$
\left\langle x^{k}\right\rangle=\frac{\partial \alpha}{\partial c_{k}}=\frac{2}{(2+k)} B_{k} c_{k}^{-k /(2+k)}>0
$$

and then, using (31) one us able to build up $I$. The general solution for $\alpha$-PDE does exist. Uniqueness is, again, proved from an analysis of the associated Cauchy problem [23].

We add now some reflections on Fisher-scaling and the virial theorem, reminding the reader that, although the relationship virial-scaling is well known (see the excellent monograph of Fernandez and Castro [24]), the novel ingredient here is Fisher's measure. Note first that Eq. (7) entails that $I$ is proportional to the expectation value of the kinetic energy of a putative physical state represented by $\psi$, a well-known fact [4]. More physical ramifications will emerge now. From (33) and (8) we have

$$
\begin{aligned}
I & =\sum_{k=1}^{M} \frac{k}{2} c_{k}\left\langle x^{k}\right\rangle=\left\langle\sum_{k=1}^{M} \frac{k}{2} c_{k} x^{k}\right\rangle= \\
& =\frac{1}{2}\left\langle x \frac{\partial}{\partial x} \sum_{k=1}^{M} c_{k} x^{k}\right\rangle=4\left\langle x \frac{\partial}{\partial x} U(x)\right\rangle,
\end{aligned}
$$

Comparing (7) and (39) we are then led to

$$
\left\langle-\frac{\partial^{2}}{\partial x^{2}}\right\rangle=\left\langle x \frac{\partial}{\partial x} U(x)\right\rangle,
$$

which can be interpreted as the quantum virial theorem for a polynomial potential of the form (8). More specifically, suppose that a physical potential $U$, after being series-expanded, is found to be well-represented by (8). Then, from scaling and the LT of $I$ one derives the virial theorem, without appealing to an equation of motion like the Schrödinger's equation.

\section{Energy eigenvalues}

Let us continue emphasizing that we have never appealed in this communication to Schrödinger's equation (SE) to derive any result. We may establish a suggestive link with it at the present stage.

From (28) and taking into account (7) we can write

$$
\alpha=-4\left\langle\frac{\partial^{2}}{\partial x^{2}}\right\rangle+\sum_{k=1}^{M} c_{k}\left\langle x^{k}\right\rangle
$$

which, using (8), can be recast as

$$
\frac{\alpha}{8}=\left\langle-\frac{1}{2} \frac{\partial^{2}}{\partial x^{2}}+U(x)\right\rangle=\langle E\rangle
$$

Then, the LT of FIM plays the role of an energy. Furthermore, the $\alpha-\mathrm{PDE}$ (36) can be recast in the fashion

$$
\frac{\alpha}{8}=\langle E\rangle=\sum_{k=1}^{M} \frac{k+2}{2} c_{k} \frac{\partial\langle E\rangle}{\partial c_{k}}
$$

The above equation can be found in Ref. [25], that deals with the WKB approach to the SE. Eq. (43) is in that reference depicted as a relationship that rules the scaling behavior of SE-energy-eigenvalues. We have thus demonstrated here, via a rather serendipitous route, that the LT of Fisher's I, given as a solution of the PDE (22), behaves under scaling in identical fashion as that of a Schrödinger energy-eigenvalue.

\section{Conclusions}

We investigated the scaling transform of Fisher's measure, in an one-dimensional scenario. Surprisingly enough, we encountered that, from the operating scaling-rules, several interesting, albeit known results can be derived, that are now seen to be pre-configured by the conjunction of scaling and information theory. See please the scheme below, that illustrates the path we followed here: 


$$
\begin{gathered}
\text { Derive Fisher's I-scaling rule } \\
\mapsto \text { manipulate it and get } \\
\mapsto \text { PDE for I. } \\
\text { Now solve PDE and study the } \\
\text { conditions that allow for a Legendre transform. } \\
\text { Apply the Legendre transform! } \\
\mapsto \text { derive associated reciprocity relations } \\
\mapsto \text { find then interesting quantum results. }
\end{gathered}
$$

In particular, we highlight that one derives in a few lines the partial differential equation that governs the FIMscaling transformation, i.e. Eq. (22). That very equation was obtained, albeit rather laboriously, in [20] from

- first appealing to the Schrödinger equation (SE) via the virial theorem and

- taking advantage afterwards of the properties exhibited by the Schrödinger equation when it is subjected to a LT.

After having gotten the FIM-solution of the scalingderived Eq. (22), one can apply a LT to its solution by changing variables (from available mean values to a new set of coefficients which can be identified as Lagrange multipliers in a MaxEnt-language or potential's seriesexpansion's coefficients in a quantum scenario). When this is accomplished, the ensuing reciprocity relations allow one to re-obtain two important quantum features

1. the virial theorem, now as a consequence (not as a pre-requisite as in [20]) and

2. the scaling behavior of the eigenvalues of the Schrödinger's equation reported Ref. [25].

Summing up, we have seen that the here advanced juxtaposition of scaling rules plus information measures (Fisher's) constitutes a rather powerful theoretical tool.

\section{Acknowledgments}

This work was partially supported by: i) the project 11/X301 of the Universidad Nacional de La Plata (UNLP), Argentine; ii) the project PIP1177 of CONICET, iii) the projects FQM-2445 and FQM-207 of the Junta de Andalucia, and iv) the project FIS2008-00781/FIS (MICINN) and FEDER (EU) (Spain, EU). AP acknowledges support from the Senior Grant CEI Bio-Tic GENIL-SPR.

\section{Appendix A: A QUANTUM RESULT}

Consider a wave function $\Psi(x)$ satisfying the Shrödinger eigenvalue equation,

$$
-\frac{\hbar^{2}}{2 m} \frac{d^{2} \Psi}{d x^{2}}+V(x) \Psi(x)=E \Psi(x),
$$

That is, $\Psi(x)$ is an eigenfunction corresponding to the potential $V(x)$ with corresponding energy eigenvalue $E$. Since the potential function $V(x)$ and the energy $E$ are real, it is clear that the complex conjugate wave function $\Psi^{*}(x)$ is also a solution of the above equation, with the same eigenvalue $E$,

$$
-\frac{\hbar^{2}}{2 m} \frac{d^{2} \Psi^{*}}{d x^{2}}+V(x) \Psi^{*}(x)=E \Psi^{*}(x) .
$$

Consequently, it follows from the linearity of the Schrödinger equation that the (real) linear combination,

$$
\Psi_{r}(x)=\frac{1}{2}\left(\Psi(x)+\Psi^{*}(x)\right),
$$

is also an eigenfunction corresponding to the potential $V(x)$ with the same eigenenergy $E$ as before. That is, for any energy eigenvalue $E$ it is always possible to find an associated real eigenfunction $\Psi_{r}(x)$. In the case of bound eigenstates of one-dimensional potentials, these real eigenfunctions are (up to an arbitrary global multiplicative phase) unique since the eigenenegies of bound eigenstates of one-dimensional potentials are nondegenerate [26]. Summing up, the eigenfunctions corresponding to bound states of one dimensional potentials can always be taken as real.

\section{References}

[1] P. W. Anderson, Science 177, 303 (1972)

[2] J. Zinn-Justin, Quantum Field Theory and Critical Phenomena (Oxford University Press, Oxford, 2002)

[3] R. N. Silver, In: Physics and Probability, W. T. Grandy Jr. and P. W. Milonni (Eds.) (Cambridge University Press, Cambridge, England, 1992)

[4] B. R. Frieden, Physics from Fisher information measure (Cambridge, University Press, Cambridge, 1998)

[5] B. R. Frieden, Science from Fisher information (Cambridge University Press, Cambidge, 2004)

[6] B. R. Frieden, A. Plastino, A. R. Plastino, B. H. Soffer, Phys. Rev. E 60, 48 (1999)

[7] S. P. Flego, B. R. Frieden, A. Plastino, A. R. Plastino, B. H. Soffer, Phys. Rev. E 68, 016105 (2003)

[8] F. Olivares, F. Pennini, A. Plastino, Physica A 389, 2218 (2010) 
[9] S. P. Flego, F. Olivares, A. Plastino, M. Casas, Entropy 13, 184 (2011)

[10] S. P. Flego, A. Plastino, A. R. Plastino, Physica A 390, 2276 (2011)

[11] M. J. W Hall, Phys. Rev. A 62, 012107 (2000)

[12] F. Pennini, A. Plastino, B. H. Soffer, C. Vignat, Phys. Lett. A 373, 817 (2009)

[13] R. Gonzalez-Ferez, J. S. Dehesa, Phys. Rev. Lett. 91, 113001 (2003)

[14] J. S. Dehesa, R. Gonzalez-Ferez, P. Sanchez-Moreno, J. Phys. A 40, 1845 (2007)

[15] A. Katz, Principles of Statistical Mechanics: The Information Theory Approach (Freeman and Co., San Francisco, 1967)

[16] E. T. Jaynes, Phys. Rev. 106, 620 (1957)

[17] H. Cramer, Mathematical methods of statistics (Princeton University Press, Princeton, NJ, 1946)

[18] C. R. Rao, Bull. Calcutta. Math. Soc 37, 81 (1945)
[19] W. Greiner and B. Mueller, Quantum mechanics. An introduction (Springer, Berlin, 1988).

[20] S. P. Flego, A. Plastino, A. R. Plastino, Physica A 390, 4702 (2011)

[21] The Schrödinger (SE) enters in [20] because, if one minimizes Fisher's I subject to constraints, one is straightforwardly led to a SE as the result of the variational process (see, for instance, [6])

[22] E. A. Deslogue, Thermal Physics (Holt, Rinehart and Winston, New York, 1968)

[23] S. P. Flego, A. Plastino, A. R. Plastino, J. Math. Phys. 52, 082103 (2011)

[24] F. M. Fernandez and E. A. Castro, Hypervirial theorems (Springer, Berlin, 1987)

[25] K. Banerjee, Proc. R. Soc. London 363, 147 (1978)

[26] R. Shankar, Principles of Quantum Mechanics (Plenum Press, New York, 1994) 